\title{
Atypical C-ANCA following high dose intravenous immunoglobulin
}

Stephen Jolles, Sarah Deacock, Wayne Turnbull, Roger Silvestrini, Chris Bunn, Peter White, Milford Ward

\section{Abstract}

Aims-(1) To assess a range of intravenous immunoglobulin products for atypical classical antineutrophil cytoplasmic antibody (C-ANCA) staining and to determine if this is present in patients treated with high dose intravenous immunoglobulin ( $2 \mathrm{~g} / \mathrm{kg} / \mathrm{month})$ and replacement doses (200 $\mathrm{mg} / \mathrm{kg}$ fortnightly); (2) using the United Kingdom national external quality assessment scheme (NEQAS), to determine if laboratories could differentiate this pattern from classical ANCA.

Methods-ANCA testing was performed on 30 batches of intravenous immunoglobulin from several manufacturers. Six patients treated with high dose intravenous immunoglobulin and 11 receiving replacement doses of immunoglobulin for hypogammaglobulinaemia were tested for ANCA by indirect immunofluorescence on cytospin preparations of ethanol fixed neutrophils and by enzyme linked immunosorbent assay (ELISA). One of the positive immunoglobulin batches was tested blindly by 125 laboratories involved in NEQAS by indirect immunofluorescence and by ELISA in some laboratories.

Results-16 of 31 batches of intravenous immunoglobulin from six different manufacturers were atypical C-ANCA positive. Three of six patients receiving high dose intravenous immunoglobulin and none of 11 patients on replacement doses were atypical C-ANCA positive. The results of the NEQAS assessment by indirect immunofluorescence were $68 \%$ C-ANCA positive, $17 \%$ negative, $9 \%$ atypical C-ANCA, and 6\% P-ANCA.

Conclusions-Some but not all intravenous immunoglobulin products yield a positive atypical cANCA by indirect immunofluorescence. An identical pattern may be observed in patients receiving high dose intravenous immunoglobulin but not in those on replacement doses. Of laboratories participating in NEQAS, $68 \%$ reported this pattern as cANCA. This reinforces the importance of reporting only "classical ANCA," defined by international ANCA workshops, to maintain the specificity of ANCA immunofluorescence and its close disease associations. (f Clin Pathol 1999;52:177-180)

Keywords: intravenous immunoglobulin; antineutrophil cytoplasmic antibodies
High dose intravenous immunoglobulin is increasingly used for its immunomodulatory properties in a wide range of inflammatory and autoimmune diseases. ${ }^{12}$ The mechanisms of action are incompletely understood and are conveniently divided into those acting through the Fc portion of the immunoglobulin molecule and those acting through the variable binding site region $\mathrm{F}(\mathrm{ab}) 2$. Those acting through $\mathrm{Fc}$ include functional blockade of reticuloendothelial system macrophages, particularly in the spleen, and inhibition of complement mediated damage by binding $\mathrm{C} 3 \mathrm{~b}$ and $\mathrm{C} 4 \mathrm{~b}$, thus reducing the number of activated complement fragments available to deposit on target surfaces. Proposed binding site $(F(a b) 2)$ interactions are neutralisation of pathogenic autoantibodies, modulation of cytokine production, selection of immune repertoires, and neutralisation of unidentified infectious agents. ${ }^{3}$

Intravenous immunoglobulin is prepared from pools of plasma from 1000-15000 healthy blood donors and therefore contains a vast range of binding specificities, making significant autoantibody titres highly unlikely. ${ }^{4}$

Antineutrophil cytoplasmic antibody (ANCA) testing by indirect immunofluorescence and enzyme linked immunosorbent assay (ELISA) is now well established in the assessment of vasculitis, ${ }^{67}$ and standardised guidelines are used..$^{8-10}$ Two main patterns are recognised-classical ANCA (C-ANCA) and perinuclear ANCA (P-ANCA). C-ANCA is associated with Wegener's granulomatosis, microscopic polyarteritis, and Churg-Strauss syndrome, ${ }^{11}$ and P-ANCA is associated with microscopic polyarteritis, Churg-Strauss syndrome, classic polyarteritis nodosa, idiopathic crescentic glomerulonephritis, systemic lupus erythematosus, and rheumatoid arthritis. ${ }^{12}$ An atypical ANCA (X-ANCA) is also recognised in association with inflammatory bowel disease, sclerosing cholangitis, autoimmune hepatitis, primary biliary cirrhosis, and connective tissue disease. ${ }^{13}$ ELISA are available for proteinase 3, which accounts for $80-90 \%$ of C-ANCA immunofluorescence, and myeloperoxidase, which accounts for $12-25 \%$ of P-ANCA. ${ }^{11}{ }^{12}{ }^{14}$ Increasing numbers of other neutrophil antigens have been defined, including bactericidal/permeability increasing protein (BPI), human elastase, lactoferrin, lysozyme, and cathepsin G, for which specific ELISA are available. ${ }^{13} 15$

Following the observation of an atypical ANCA in a patient receiving high dose
Accepted for publication 10 November 1998 
Table 1 Methodology and results of analysis of intravenous immunoglobulin preparations by immunofluorescence (NEQAS analyses)

\begin{tabular}{lllll}
\hline IF method & C-ANCA & P-ANCA & Negative & Atypical \\
\hline In-house & 28 & 2 & 7 & 4 \\
Inova & 15 & 1 & 2 & 3 \\
Biodiagnostics & 11 & 0 & 4 & 1 \\
Binding Site & 21 & 2 & 3 & 2 \\
Euroimmun & 1 & 0 & 0 & 0 \\
Immco & 3 & 1 & 1 & 0 \\
CLS & 0 & 1 & 0 & 0 \\
Not stated & 2 & 0 & 3 & 1 \\
Total & 81 & 7 & 20 & 11 \\
\hline
\end{tabular}

C-ANCA, classical antineutrophil cytoplasmic antibody; IF, immunofluorescence; P-ANCA, perinuclear ANCA.

intravenous immunoglobulin, which was also present in the immunoglobulin preparation, we decided to investigate patients on a range of intravenous immunoglobulin products for this pattern. The aim was to establish which products were positive and the numbers of patients who became positive after high dose compared with replacement doses of intravenous immunoglobulin. In addition the ability of United Kingdom immunology laboratories to distinguish the atypical pattern from classical ANCA was assessed by sending a positive intravenous immunoglobulin sample to laboratories participating in the national external quality assessment scheme (NEQAS).

\section{Methods}

Indirect immunofluorescence screening of all serum and intravenous immunoglobulin was performed using a 1:10 dilution. Samples were applied to cytospin preparations of ethanol

Table 2 Methodology and results of analysis of intravenous immunoglobulin preparations by enzyme linked immunosorbent assay (NEQAS analyses)

\begin{tabular}{llllll}
\hline ELISA method & PR3 & Equivocal PR3 & MPO & Equivocal MPO & Negative \\
\hline In-house & 1 & 0 & 4 & 0 & 2 \\
Diastat & 1 & 0 & 1 & 0 & 8 \\
Wieslab & 1 & 0 & 0 & 0 & 4 \\
Biodiagnostics & 0 & 2 & 1 & 2 & 4 \\
Genesis & 1 & 1 & 1 & 0 & 0 \\
Inova & 0 & 1 & 0 & 0 & 4 \\
Eurodiagnostics & 0 & 0 & 0 & 0 & 1 \\
F A Elias & 3 & 0 & 0 & 0 & 0 \\
Progen & 0 & 0 & 0 & 0 & 1 \\
Not stated & 0 & 1 & 0 & 0 & 28 \\
Total & 7 & 5 & 7 & 2 & \\
\hline
\end{tabular}

ELISA, enzyme linked immunosorbent assay; MPO, myeloperoxidase; PR3, proteinase 3.

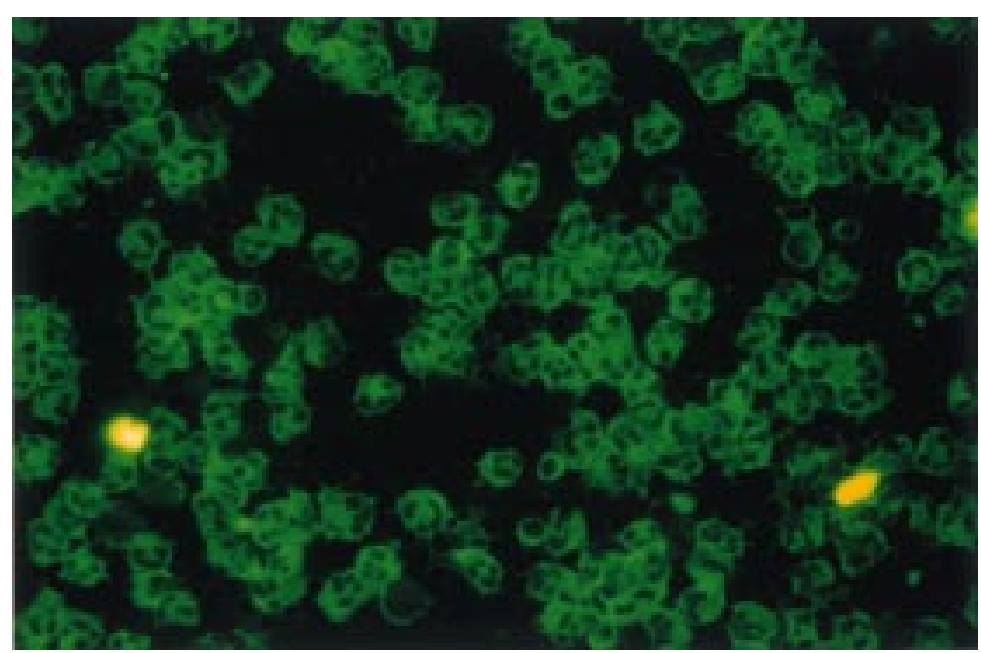

Figure 1 Indirect immunofluorescence of neutrophils to intravenous immunoglobulin. fixed neutrophils. ${ }^{9}$ Incubation of these cells with serum or intravenous immunoglobulin was followed by a wash step and the addition of a fluorescein conjugated antihuman IgG (Dako). Following a further wash step the neutrophils were viewed at $\times 400$ magnification on a Zeiss fluorescence microscope.

ELISA for proteinase 3 and myeloperoxidase were performed according to the Shield Diagnostics Diastat and ORGanTec kit protocols.

The methods used in the NEQAS analysis are shown separately in tables 1 and 2 .

\section{Results}

Six patients were treated with high dose intravenous immunoglobulin for the following conditions: atopic dermatitis (1), dermatomyositis (1), pemphigus vulgaris (1), epidermolysis bullosa acquisita (1), and Guillain-Barré syndrome (2). Intragam was given for the patients with Guillain-Barré syndrome and Sandoglobulin for the remainder, at a dose of $2 \mathrm{~g} / \mathrm{kg}$. ANCA testing was performed before and after the treatment. Three patients became atypical C-ANCA positive after treatment. The titre of this pattern was usually less than 1/160 and became negative in two of the three patients after four weeks, just before the next treatment cycle.

The batches of immunoglobulin used were all positive for atypical C-ANCA, with titres of up to $1 / 320$. Serial neutrophil counts were also performed and fell transiently in the atypical ANCA positive epidermolysis bullosa patient to $0.5 \times 10^{9} / \mathrm{mm}^{3}$, recovering to normal within one week; they remaining stable in the other patients.

The immunofluorescence pattern of the intravenous immunoglobulin, and a known C-ANCA, are shown in figs 1 and 2, respectively. The intravenous immunoglobulin shows a "flat" ANCA pattern, without the central or interlobular accentuation seen in classical ANCA. Lymphocyte staining (and eosinophil staining, not shown) was also observed, which is not seen in classical ANCA. Proteinase 3 and myeloperoxidase ELISA were negative in two of the "flat" ANCA patients and proteinase 3 was positive in the third (ORGanTec ELISA).

We tested a range of intravenous immunoglobulin batches from different manufacturers (Sandoglobulin, Bern, Switzerland; Gammimune N, Spokane, Washington, USA; Alphaglobin, Barcelona, Spain; Endobulin, Vienna, Austria; Octogam, Vienna, Austria; Intragam and CPIVIG, Central Serum Laboratories, Melbourne, Australia) and showed a positive atypical C-ANCA in 11/11 Sandoglobulin, 1/1 Gammimune N, 3/3 Intragam, 1/1 CPIVIG; with 10/10 Alphaglobin, 1/1 Octagam, and $4 / 4$ Endobulin ANCA negative, even though the negatives were brighter than negative controls. All intravenous immunoglobulin was assessed at a concentration of $3 \% \quad(1 / 10$ dilution). Eleven patients with primary immunodeficiencies receiving regular replacement doses of intravenous immunoglobulin (Sandoglobulin), $0.2 \mathrm{~g} / \mathrm{kg}$ every two weeks, were ANCA negative. This measurement was made just 


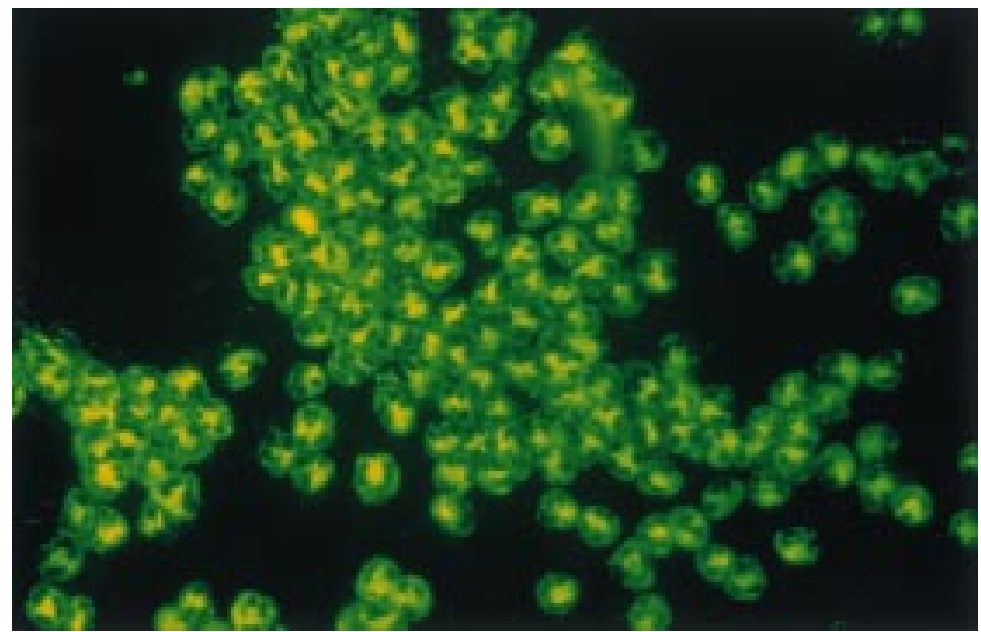

Figure 2 Indirect immunofluorescence of neutrophils to a known positive C-ANCA control. before the next immunoglobulin infusion and thus represented trough level values, which are maintained at more than $8 \mathrm{~g} /$ litre in our unit.

To assess the interpretation of an atypical C-ANCA positive batch of intravenous immunoglobulin, a sample was sent to NEQAS. This sample was distributed to 134 laboratories and of 119 returns, 81 reported C-ANCA, seven P-ANCA, 20 negative, and 11 atypical ANCA. The results from the laboratories which carried out ELISA were as follows: seven proteinase 3 positive, five proteinase 3 equivocal, seven myeloperoxidase positive, two myeloperoxidase equivocal, and 28 negative. The results for both immunofluoresence and ELISA are shown in figs 3 and 4.

\section{Discussion}

The results show that the atypical C-ANCA immunofluorescence found in intravenous immunoglobulin occurs after high dose intravenous immunoglobulin treatment and may be confused with classical ANCA, as shown by the NEQAS data in which $68 \%$ of laboratories reported the pattern as unequivocal C-ANCA positive. The positive ELISA results are more difficult to explain in view of the high specificity of the assays. However, many more of the ELISA gave negative results $(58 \%)$ than

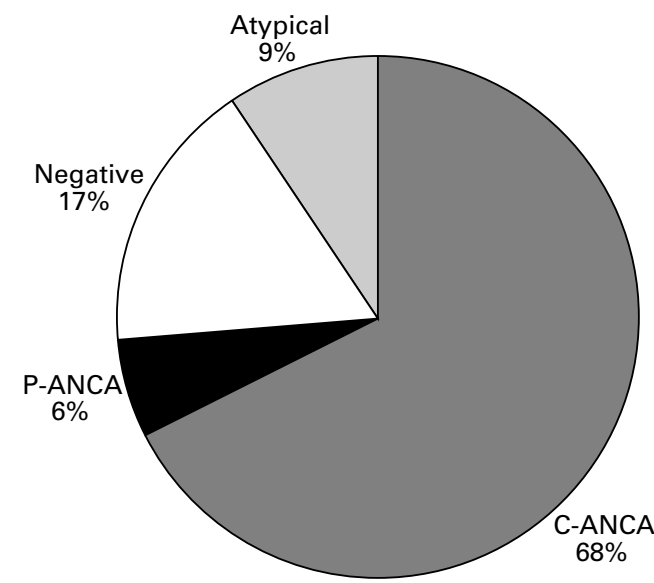

Figure 3 Intravenous immunoglobulin sample analysed by immunofluorescence.

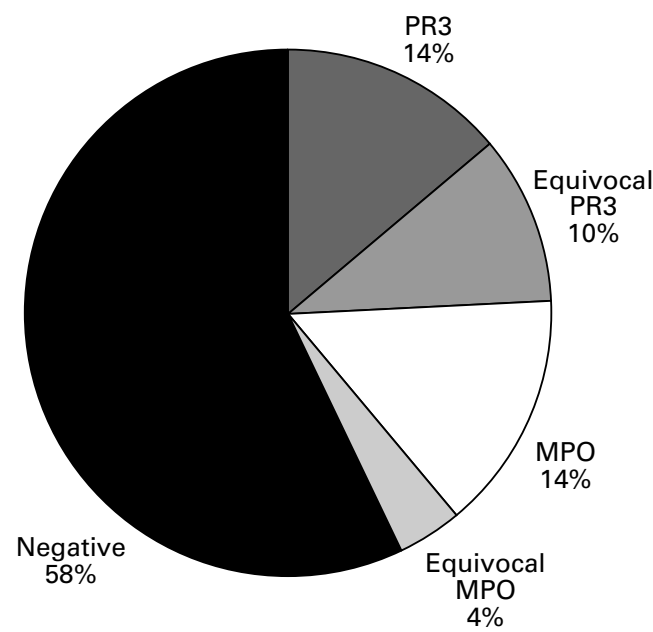

Figure 4 Intravenous immunoglobulin sample analysed by enzyme linked immunosorbent assay.

did immunofluorescence (17\%), confirming the usefulness of using immunofluorescence and ELISA in combination for difficult cases. Furthermore, it seems unlikely that when extensively screened healthy donors are used to manufacture immunoglobulin preparations there would be sufficient ANCA to explain these results. Other autoantibodies - for example, antinuclear, anti-gliadin, and antiendomysial antibodies, which are more common in the general population than ANCAare not found in patients treated with intravenous immunoglobulin.

The atypical ANCA pattern seen may be distinguished by its finer granularity, lack of central and interlobular accentuation resulting in the "flat" appearance, and staining of lymphocytes (and eosinophils) when present in the preparation. Some contamination with lymphocytes is helpful in distinguishing the patterns.

Binding of intravenous immunoglobulin to other neutrophil antigens, for example elastase, lactoferrin, lysozyme, BPI, or cathepsin G, has not yet been established. The effect may be non-specific in nature-perhaps related to differences in the manufacturing process - and the clear differences observed between the different manufacturers lends support to a production dependent mechanism. However, although heat inactivation of serum may result in an artefactual ANCA pattern, all the Alphaglobin batches tested, which are treated by pasteurisation at $60^{\circ} \mathrm{C}$, were ANCA negative. Alternatively, binding of intravenous immunoglobulin may occur through Fc and complement receptors or it may be that the high protein content could interfere with the assays.

There are no previous reports of atypical C-ANCA following high dose intravenous immunoglobulin. However, one case report, which describes uveitis associated with ANCA positivity following intravenous immunoglobulin at replacement doses, suggested a pathogenic role for the ANCA observed. ${ }^{16}$ Antineutrophil antibodies and transient neutropenia following intravenous immunoglobulin treatment have also been also described. ${ }^{17} 18$ 
CONCLUSION

This is the first report of ANCA positivity following high dose intravenous immunoglobulin, and it is important for laboratories to be aware of this phenomenon in order to be able to offer clinically useful advice. Information about concurrent intravenous immunoglobulin treatment on the request form would facilitate appropriate reporting, particularly as high dose intravenous immunoglobulin may be used in the treatment of vasculitis. ${ }^{19}$ In addition, the atypical nature of the ANCA pattern observed and the confusion regarding the immunofluorescence results reinforces the importance of reporting only "classical ANCA" (defined by international ANCA workshops ${ }^{8-10}$ ) as C-ANCA rather than other forms of cytoplasmic staining. This would avoid diagnostic confusion and maintain the specificity of ANCA immunofluorescence and its close disease associations.

1 Dwyer JM. Manipulating the immune system with immune globulin. N Engl f Med 1992;326:107-16.

2 Jolles S, Hughes JR, Whittaker SJ. Dermatological uses of high dose intravenous immunoglobulin (hdIVIg). Arch high dose intravenous im

3 Mouthon SV, Kaveri SH, Spalter S, et al. Mechanisms of action of intravenous immune globulin in immune mediated diseases. Clin Exp Immunol 1996;104(suppl 1):310.

4 Rutter GH. Requirements for safety and quality of intravenous immunoglobulin $G$ preparations. $\mathcal{F}$ Neurol Neurosurg Psychiatry 1994;57(suppl):2-5.

5 Mouthon L, Kazatchkine MD. Autoantibodies in therapeutic preparations of human IgG (IVIg). In: Peter JB, Schoenfeld Y, eds. Autoantibodies. Amsterdam: Elsevier Science, 1996:91-6.
6 Roberts DE. Antineutrophil cytoplasmic antibodies. Clin Lab Med 1992;12:85-98.

7 Savage COS, Harper L, Adu D. Primary systemic vasculitis. Lancet 1997;349:553-8.

8 Rasmussen N, Wiik A. Indirect immunofluorescence examination for IgG-ANCA in sera submitted for the 1st international workshop on ANCA, 1988. APMIS 1989; 97(suppl 6):16-20.

9 Lock RJ. ACP Broadsheet No 143: Detection of autoantibodies to neutrophil cytoplasmic antigens. 7 Clin Pathol 1994;47:4-8.

10 Wiik A. Delineation of a standard procedure for indirect immunofluorescence detection of ANCA. APMIS 1989; 97(suppl 6): 12-13.

11 Gross WL, Csernok E, Szymkowiak CH. Antineutrophil cytoplasmic antibodies with specificity for proteinase 3. In: Peter JB, Schoenfeld Y, eds. Autoantibodies. Amsterdam: Elsevier Science, 1996:61-8.

12 Kallenberg CGM. Antineutrophil cytoplasmic antibodies with specificity for myeloperoxidase. In: Peter JB, Schoenfeld Y, eds. Autoantibodies. Amsterdam: Elsevier Science, 1996:53-61.

13 Zhao MH, Lockwood CM. Antineutrophil cytoplasmic antibodies with specificity other than proteinase 3 and antibodies with specificity other than proteinase 3 and
myeloperoxidase (X-ANCA). In: Peter JB, Schoenfeld Y, eds. Autoantibodies. Amsterdam: Elsevier Science, 1996: eds. Avtrod 68 .

14 Jennings JG, Chang L, Savige JA. Anti-proteinase 3 antibodies, their characterization and disease associations. Clin Exp Immunol 1994;95:251-6.

15 Zhao MH, Jones SJ, Lockwood CM. Bactericidal/ permeability-increasing protein (BPI) is an important antigen for anti-neutrophil cytoplasmic antibodies in vasculitis. Clin Exp Immunol 1995;99:49-56.

16 Ayliffe W, Haeney M, Roberts SC, et al. Uveitis after antineutrophil cytoplasmic antibody contamination of immunoglobulin replacement therapy [letter]. Lancet 1992;339:558-9.

17 Ben-Chetrit E, Putterman C. Transient neutropenia induced by intravenous immune globulin [letter]. $N$ Engl $\mathcal{F}$ Med 1992:326:270-1.

18 Lassiter HA, Bibb KW, Bertolone SJ, et al. Neonatal immune neutropenia following the administration of immune globulin. Am f Pediatr Hematol Oncol 1993;15: $120-3$.

19 Lockwood CM. New treatment strategies for systemic vasculitis: the role of intravenous immune globulin therapy. Clin Exp Immunol 1996;104(suppl 1):77-83. 